\title{
Enhanced Skin Delivery of Genistein and Other Two Isoflavones by Microemulsion and Prevention against UV Irradiation-Induced Erythema Formation
}

\author{
Shuji Kitagawa, ${ }^{*}$ Kana Inoue, Reiko Teraoka, and Shin-ya Morita \\ Kobe Pharmaceutical University; 4-19-1 Motoyamakita-machi, Higashinada-ku, Kobe 658-8558, Japan. \\ Received September 17, 2009; accepted December 16, 2009
}

To improve the efficiency of the intradermal delivery of genistein and other two isoflavones (daidzein and biochanin A), we tried to clarify the usefulness of microemulsion by in vitro study on excised guinea pig dorsal skin and Yucatan micropig skin. Using microemulsion consisting of isopropyl myristate (IPM), $150 \mathrm{~mm} \mathrm{NaCl} \mathrm{so-}$ lution, Tween 80 and ethanol as a vehicle, the solubility of all the isoflavones markedly increased and significant amounts of isoflavones were delivered to the skin. The effect of water-in-oil (w/o)-type microemulsion D was larger than that of oil-in-water (o/w)-type microemulsion A. Among three isoflavones tested, the increase of genistein was most marked on both solubility and skin accumulation. Genistein retained in the skin significantly inhibited lipid peroxidation in vitro dose-dependently. Furthermore, pretreatment of guinea pig dorsal skin with genistein containing gel-like microemulsion D prevented UV irradiation-induced erythema formation. These findings indicate the potential use of w/o-type microemulsion for the delivery of genistein to protect skin against UV-induced oxidative damage.

Key words isoflavone; genistein; skin; intradermal delivery; microemulsion; UV irradiation

Polyphenols such as flavonoids are known as antioxidants and have been applied for topical purposes such as photoprotection against UV-induced skin damage (photoaging), skin cancer prevention, and skin care ${ }^{1-3)}$; however, intradermal delivery of most polyphenols is inefficient, due to their low skin permeability as well as their low solubility in both aqueous and organic media. Systems should therefore be improved to enable their efficient incorporation. To this end, the use of prodrugs, ${ }^{4)}$ chemical enhancers, ${ }^{5)}$ and liposomes ${ }^{6)}$ has been examined for the skin delivery of various polyphenols.

For this purpose we used a microemulsion as an enhancement system, which consisted of an aqueous phase, an organic phase, a surfactant and a co-surfactant component. The microemulsion is thermodynamically stable and has been shown to have high solubilization capacity and to facilitate the skin permeation of both lipophilic and hydrophilic drugs. ${ }^{7,8)}$ Although microemulsion has not been widely used for skin absorption of polyphenols, recently, the enhancement effect of a microemulsion on the intradermal delivery of quercetin was reported by Vicentini et al., ${ }^{9)}$ in which a water-in-oil (w/o) microemulsion consisting of propylene glycol, water, Span 80, and canola oil was used. We also reported the usefulness of w/o-type microemulsion consisting of isopropyl myristate (IPM), $150 \mathrm{~mm} \mathrm{NaCl}$ solution, Tween 80 and ethanol to improve the efficiency of intradermal delivery of quercetin. ${ }^{10)}$

In this study we tried to clarify the usefulness of the same microemulsion for intradermal delivery of genistein and other two isoflavones, shown in Fig. 1. Although it was revealed that genistein prevented UV-light-induced signaling that leads to photoaging, ${ }^{11)}$ the intradermal delivery as well as solubility of genistein is known to be very limited. To improve intradermal delivery we used a microemulsion in this study. We compared the effects of the microemulsion on skin accumulation as well as the solubility of each isoflavone. We also examined the usefulness of the retained isoflavones to prevent lipid peroxidation in skin as an in vitro study. To

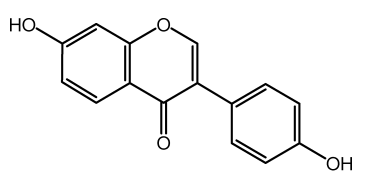

a
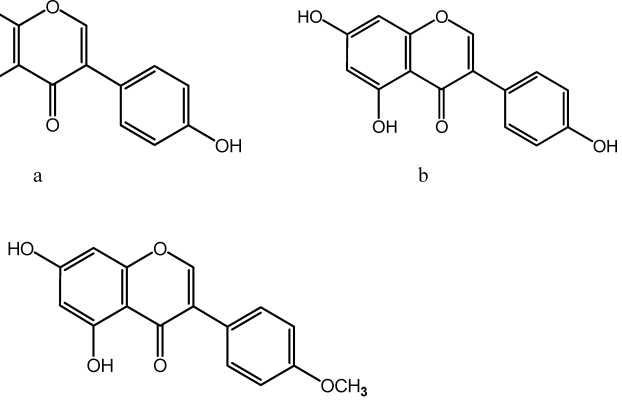

c

Fig. 1. Isoflavones Tested in This Study

(a) Daidzein, (b) genistein, (c) biochanin A.

reveal the effects of isoflavone application, which showed most marked skin accumulation, on photoaging of skin, we furthermore examined the effects of topical application of the isoflavone with gel-like microemulsion using guinea pigs on ultraviolet light-induced erythema formation in vivo.

\section{Results and Discussion}

We first examined the effects of oil-in-water (o/w)-type microemulsion A and w/o-type microemulsion D on the solubility of three isoflavones, daidzein, genistein and biochanin A, whose logarithm values of partition coefficients between $n$-octanol and phosphate buffered saline (PBS) were 1.93, 2.75 and 3.14 , respectively. The mean particle diameter of microemulsion $\mathrm{A}$ and $\mathrm{D}$ was $39.8 \pm 22.8 \mathrm{~nm}$ and $42.5 \pm 21.5$ $\mathrm{nm}$, respectively. ${ }^{10)}$ As shown in Table 1 , the solubility of isoflavones in $150 \mathrm{~mm} \mathrm{NaCl}$ and that in IPM was very limited. Both microemulsions significantly improved the solubility of isoflavones, in the order of genistein $>$ biochanin $\mathrm{A}>$ daidzein. The increase with microemulsion $\mathrm{D}$ was larger than that with microemulsion A.

As shown in Table 2 for the results on excised guinea pig skin, the intradermal delivery of all three isoflavones was 
Table 1. Effects of Microemulsions on Solubility of Isoflavones

\begin{tabular}{llll}
\hline \hline \multirow{2}{*}{ Vehicle } & \multicolumn{3}{c}{ Solubility (mM) } \\
\cline { 2 - 4 } & \multicolumn{1}{c}{ Daidzein } & Genistein & Biochanin A \\
\hline $150 \mathrm{~mm} \mathrm{NaCl}$ & $0.029 \pm 0.003$ & $0.059 \pm 0.011$ & $0.718 \pm 0.003$ \\
$\mathrm{IPM}$ & $0.072 \pm 0.009$ & $0.959 \pm 0.097$ & $6.21 \pm 0.69$ \\
Microemulsion A & $11.46 \pm 0.55^{* * *}$ & $80.27 \pm 4.63^{* * *}$ & $39.37 \pm 2.87 * * *$ \\
Microemulsion D & $18.53 \pm 0.30^{* * *}$ & $140.5 \pm 13.1 * * *$ & $87.58 \pm 6.61 * *$ \\
& & & \\
\hline
\end{tabular}

Data are the means \pm S.D. of four experiments. $* * * p<0.001$, significantly different from the values in $\mathrm{NaCl}$ solution and IPM.

Table 2. Effects of Microemulsions on Intradermal Delivery of Isoflavones

\begin{tabular}{lclc}
\hline \hline \multirow{2}{*}{ Vehicle } & \multicolumn{2}{c}{ Skin accumulation $(\mu \mathrm{mol} / \mathrm{g}$ skin $)$} \\
\cline { 2 - 4 } & Daidzein & Genistein & Biochanin A \\
\hline $150 \mathrm{mM} \mathrm{NaCl}$ & $0.37 \pm 0.14$ & $0.10 \pm 0.01$ & $0.19 \pm 0.05$ \\
$\mathrm{IPM}$ & $0.26 \pm 0.24$ & $0.25 \pm 0.05$ & $0.30 \pm 0.07$ \\
Microemulsion A & n.d. & $3.55 \pm 0.60^{* * *}$ & n.d. \\
Microemulsion D & $2.29 \pm 0.32^{* * *}$ & $5.92 \pm 1.48^{* * *}$ & $1.30 \pm 0.37 * * *$ \\
Microemulsion D\# & n.d. & $2.58 \pm 0.55^{* * *}$ & n.d. \\
\hline
\end{tabular}

Data are the means \pm S.D. of three experiments. $* * * p<0.001$, significantly different from the values in $\mathrm{NaCl}$ solution and IPM. D\#, gel-like microemulsion D. n.d., no data.

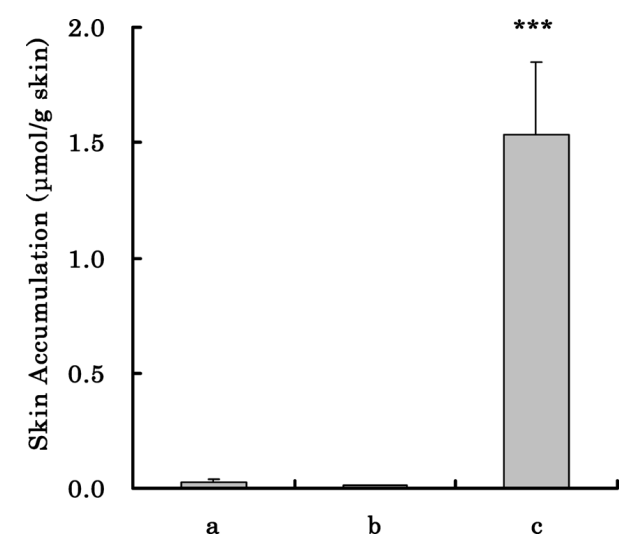

Fig. 2. Skin Accumulation of Genistein in Yucatan Micropig Skin when Applied as a Suspension in Either $150 \mathrm{~mm} \mathrm{NaCl}$ Solution (a) or IPM (b) and Microemulsion D at Saturated Concentration (140 mM) (c)

Data are the means \pm S.D. of four experiments. $* * * p<0.001$, significantly different from the values in $\mathrm{NaCl}$ and IPM.

markedly increased by using w/o-type microemulsion $\mathrm{D}$ as a vehicle, compared with the delivery when either $150 \mathrm{~mm}$ $\mathrm{NaCl}$ or IPM was used as a vehicle. Skin accumulation was in the order of genistein $>$ daidzein $>$ biochanin A. Since the increase using microemulsion D was largest for genistein both in solubility and skin accumulation among the three isoflavones, we also examined the effect of microemulsion A on the topical delivery of genistein. As also shown in Table 2 , the accumulation of genistein with microemulsion A was much larger than that either with $150 \mathrm{~mm} \mathrm{NaCl}$ or IPM, but was less that with microemulsion D. Gel-like microemulsion prepared by the addition of $8.3 \%$ AEROSIL R972 to microemulsion D also increased the skin accumulation, although it decreased to $44 \%$ of that without AEROSIL.

Enhancement by microemulsion was also confirmed when Yucatan micropig skin, which is hairless and has been suggested to show physiological similarity to human skin, ${ }^{12)}$ was

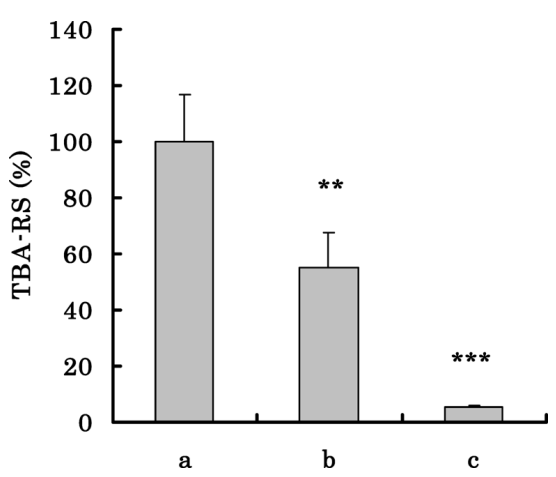

Fig. 3. Effect of Genistein on Lipid Peroxidation Tested by Ammonium Iron(II) Sulfate and Sodium Citrate When Applied with Microemulsion D at $0 \mathrm{~mm}$ (a), $20 \mathrm{~mm}$ (b) or Saturated Concentration (140 mм) (c)

Data are the means \pm S.D. of four experiments. $* * p<0.01$, $* * * p<0.001$, significantly different from the value in the absence of genistein.

(a)

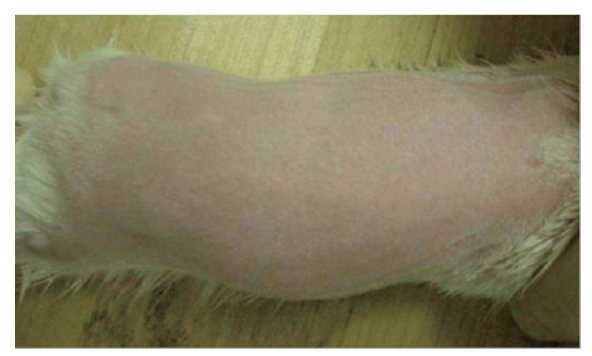

(b)

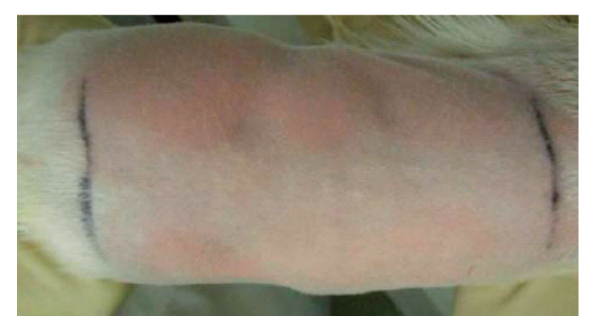

Fig. 4. Effect of Genistein on UV-B-Induced Erythema Formation (a) before UV Irradiation (b) after UV Irradiation

Upper part: gel-like microemulsion D without genistein prepared by $8.3 \%$ AEROSILR 972 was applied. Lower part: Gel-like microemulsion D containing $140 \mathrm{~mm}$ genistein was applied $20 \mathrm{~h}$ before UV irradiation.

used with genistein applied with microemulsion $\mathrm{D}$, as shown in Fig. 2.

To know the antioxidative effects of isoflavones incorporated into the skin using microemulsion as a vehicle, the antilipoperoxidative activity of genistein, whose efficiency in skin incorporation was highest among the three isoflavones tested, retained in the skin was estimated by the formation of malondialdehyde (MDA) using iron(II) and citrate. ${ }^{13)}$ As shown in Fig. 3, genistein retained in the skin significantly inhibited lipid peroxidation in a dose-dependent manner.

We furthermore examined the effects of topical application of genistein on guinea pig with gel-like microemulsion, which was prepared with $8.3 \%$ AEROSIL R972, on UVinduced erythema formation. UV light was irradiated at an intensity of $138 \mu \mathrm{W} / \mathrm{cm}^{2}$ for $24 \mathrm{~min}$. As shown in Fig. 4, preloading of $140 \mathrm{~mm}$ genistein with gel-like microemulsion D significantly inhibited erythema formation. Redness increase 
in skin measured with a color reader, which was expressed as the $a^{*}$ difference values on the skin surface before and after irradiation, was $4.5 \pm 1.7$ in control skin and $1.0 \pm 0.7 * *$ in skin pretreated with genistein $(n=6, * * p<0.01)$.

Microemulsions offer several advantages for pharmaceutical use, such as long-term stability, high solubilization capacity for both hydrophilic and lipophilic drugs, and improved dermal and transdermal drug delivery. ${ }^{8)}$ Microemulsions consist of an oil phase and aqueous phase as well as a surfactant and co-surfactant. As surfactants, various compounds, such as polysorbates, polyoxyethylene alkyl ethers and phospholipids, are used, and short chain alcohols are often used as co-surfactants. ${ }^{8,14)}$ The findings obtained in this study also revealed that the microemulsion, which consisted of $150 \mathrm{~mm}$ $\mathrm{NaCl}$ solution, IPM, Tween 80 and ethanol, markedly improved the intradermal delivery of isoflavones as well as their solubility. A microemulsion is therefore of benefit for the skin delivery of isoflavones, which has very low solubility in both aqueous and non-aqueous vehicles. These findings are consistent with recent findings on quercetin. ${ }^{10)}$ Both the solubility and skin accumulation of genistein revealed in this study were greater than those of quercetin (solubility 78.6士 $8.9 \mathrm{~mm}$, skin accumulation $3.66 \pm 0.27 \mu \mathrm{mol} / \mathrm{g}$ skin in microemulsion D). The increase in solubility with w/o-type microemulsion D was more marked than that with o/w-type microemulsion A, which is probably related to the hydrophobicity of genistein whose logarithm value of partition coefficient between $n$-octanol and PBS was 2.75. These findings are characteristic of microemulsion because, in general, the increased solubility of a solute in the vehicle increases its thermodynamic stability in the vehicle, which leads to decreased partition in the skin. As a result, increased solubility does not usually lead to increased intradermal or transdermal drug absorption.

The increase in intradermal delivery was in the order of genistein $>$ daidzein $>$ biochanin $A$. Since the order did not agree with the order of partition coefficients, which were biochanin A (logarithm value of partition coefficient was 3.14) $>$ genistein (2.75) $>$ daidzein (1.93), the presence of an $\mathrm{OH}$ group in the B-ring may also affect the interaction with microemulsion as suggested for flavonoid-protein interaction. $^{15)}$

Since enhancement by microemulsion was also confirmed when Yucatan micropig skin, which is hairless, was used, enhancement was induced in the penetration process through the stratum corneum. Although the mechanism of the enhanced delivery of isoflavones by microemulsion is not clear, one possibility is as follows: microemulsion vehicles break down when they come into contact with the skin, releasing isoflavones, which is expected to be mainly present in the interface region of the microemulsion, into the skin and stimulating its delivery into the surface of the stratum corneum. ${ }^{7}$ A microemulsion may also enhance the skin delivery of isoflavones partly by mixing its components with lipid lamella of the stratum corneum and enhancing the permeation of isoflavones through the stratum corneum. In addition, continuously and spontaneously fluctuating interfaces of microemulsions enable high drug mobility and might enhance the drug diffusion process. ${ }^{16)}$

UV-B, which is an essential component of sunlight, crosses the epidermis and reaches the upper dermis, induces erythema formation, premature skin aging, inflammation and photocarcinogenesis, probably through the formation of reactive oxygen species. ${ }^{9,17)}$ In the present study we revealed that genistein inhibited lipid peroxidation in guinea pig dorsal skin tested by iron(II) and citrate. We furthermore revealed that genistein inhibited UV-B-induced erythema formation. The results are different from those by Kang et al., ${ }^{11)}$ who found no effect of genistein on UV-B-induced erythema formation. This is possibly due to the difference of the vehicle and skin accumulation amount of genistein as used $70 \%$ ethanol, 30\% propylene glycol and $0.05 \%$ 3,5-di-tert-butyl4-hydroxytoluene. ${ }^{11)}$ Since the use of microemulsion markedly increased the skin accumulation of genistein, it probably showed a protective effect on UV-B-induced erythema formation. This protective effect is possibly due to the reduction of reactive oxygen species by genistein as suggested by Casagrande et al. for the effect of quercetin. ${ }^{12,18)}$

The findings in this study indicate the potential use of microemulsion, especially w/o-type microemulsion for the efficient delivery of isoflavones, especially genistein, to protect skin against UV-induced oxidative damage, presumably by its antioxidant activity.

\section{Experimental}

Materials Genistein, daidzein and biochanin A were purchased from Funakoshi Co. (Tokyo, Japan). Polyoxyethylene sorbitan monooleate (Tween 80) and isopropyl myristate (IPM) were obtained from Nacalai Tesque (Kyoto, Japan). Ethanol and all other reagents were purchased from Wako Pure Chemical Industries (Osaka, Japan). AEROSIL R972 was offered by NIPPON AEROSIL Co., Ltd. (Tokyo, Japan). Dorsal skin was excised from guinea pigs following the protocol approved by the Animal Experimentation Committee of Kobe Pharmaceutical University. Pentobarbital sodium was used for anesthesia. Subcutaneous fat and other extraneous tissues were trimmed. Yucatan micropig skin (YMP skin set) was purchased from Charles River Japan (Yokohama, Japan). The fat and subdermal tissue were removed following the method of Fujii et al. ${ }^{19)}$

Preparation of Microemulsion Microemulsion A (o/w-type) and D (w/o-type) were prepared using $150 \mathrm{~mm} \mathrm{NaCl}$ solution as an aqueous phase and isopropyl myristate (IPM) as an oil phase, Tween 80 as a surfactant, and ethanol as a co-surfactant, by modifying the method of Lee et al. $^{20)}$ Weight ratio of IPM, $\mathrm{NaCl}$ solution, Tween 80 and ethanol was $8: 25: 20: 47$ in microemulsion A, and $33: 7: 30: 30$ in microemulsion D. Ethanol also works as an ingredient in the aqueous phase. Each microemulsion was obtained by vortex mixing for a few minutes at $37^{\circ} \mathrm{C}$.

Measurement of Intradermal Delivery In vitro study on the skin incorporation of isoflavones was examined as described previously. ${ }^{10,21)}$ Guinea pig dorsal skin or Yucatan micropig skin was mounted in a Franz cell with a water jacket $\left(37^{\circ} \mathrm{C}\right)$. The available diffusion area was approximately $0.71 \mathrm{~cm}^{2}$ and the receptor cell had a capacity of about $4.7 \mathrm{ml}$. After $12 \mathrm{~h}$ pretreatment of the skin and washing both donor and receptor compartments, $1 \mathrm{ml}$ microemulsion containing isoflavones, which were solubilized by addition to the microemulsion, was added to the donor compartment while phosphate-buffered saline (PBS) was added to the receptor compartment. Gel-like microemulsion was prepared by adding AEROSIL R972 to the microemulsion to $8.3 \%$ with stirring, and added to donor compartments similarly. After $20 \mathrm{~h}$ treatment ( $40 \mathrm{~h}$ treatment for Yucatan micropig skin), at which the amount of isoflavones penetrating the skin was close to the maximum level, the skin was removed from the cell, the treated area was punched out and washed with ice-cold methanol. After drying at ambient temperature, the skin was weighed (about $0.09 \mathrm{~g}$ for guinea pig skin), minced and placed in $10 \mathrm{ml}$ methanol, and then homogenized using a tissue homogenizer Polytron PT3100 (Kinematica, Luzern, Switzerland) at $5000 \mathrm{rpm}$ for $1 \mathrm{~min}$. The samples were then centrifuged and the supernatant layer was used to determine the concentration of isoflavones by HPLC LC-10-AS (Shimadzu, Kyoto, Japan). Separation was achieved on a reversed-phase column (Mightysil RP-18 GP, $4.6 \mathrm{~mm}$ i.d., $150 \mathrm{~mm}$ ) using a mobile phase consisting of methanol, water and phosphoric acid $(100: 100: 1)$. SPD-10AVvp (Shimadzu, Kyoto, Japan) was used as a UV detector. The detection wavelengths were $260 \mathrm{~nm}$ for genistein and biochanin A, and $250 \mathrm{~nm}$ for daidzein. As an 
internal standard, trans-ferulic acid was used for all isoflavones.

Measurement of Solubility Isoflavone solubility was measured after incubation of excess amount of isoflavone in either microemulsion, IPM or $150 \mathrm{~mm} \mathrm{NaCl}$ solution at $37^{\circ} \mathrm{C}$ for about $20 \mathrm{~h}$. After quick centrifugation at $12000 \times \boldsymbol{g}$ for $1 \mathrm{~min}$, the concentration of the supernatant was determined by HPLC as described above.

Measurement of Partition Coefficients of Isoflavones Partition coefficients of isoflavones between $n$-octanol and PBS were measured as described previously. ${ }^{22)}$ PBS ( $\left.3 \mathrm{ml}\right)$ was mixed with $3 \mathrm{ml} n$-octanol, which contains isoflavones $(0.1-1.0 \mathrm{~mm})$, in test tubes with glass stoppers. The PBS and $n$-octanol solutions were pre-saturated with either $n$-octanol or PBS and deoxygenized with a nitrogen stream. The test tubes were set at $37^{\circ} \mathrm{C}$ for $18 \mathrm{~h}$ in a shaking water bath. After shaking, incubation continued for another $1 \mathrm{~h}$. The concentration of isoflavones in both the PBS phase and the $n$ octanol phase was determined by HPLC as described above.

Measurement of Lipid Peroxidation The antilipoperoxidative activity of isoflavones retained in the skin was estimated by the formation of malondialdehyde (MDA) tested using iron(II) and citrate. ${ }^{13)}$ Skin was homogenized in $1.15 \% \mathrm{KCl}$ solution. To $1.0 \mathrm{ml}$ of each sample containing about $1 \mathrm{mg}$ protein, ammonium iron(II) sulfate and sodium citrate were added to a final concentration of $50 \mu \mathrm{M}$ and $2 \mathrm{~mm}$, respectively, and kept for $30 \mathrm{~min}$ at $37^{\circ} \mathrm{C}$. To determine MDA formation, $0.20 \mathrm{ml}$ of $8.1 \%$ sodium dodecyl sulfate solution, $1.5 \mathrm{ml}$ acetate buffer adjusted to $\mathrm{pH} 3.5,50 \mu \mathrm{l}$ of $0.8 \%$ butylhydroxytoluene (BHT) in glacial acetic acid, $1.5 \mathrm{ml}$ of $0.8 \%$ thiobarbituric acid (TBA) solution, and $0.7 \mathrm{ml}$ of $5 \mathrm{~mm}$ iron(III) chloride solution, were further added in this order, followed by 60 -min incubation at $60^{\circ} \mathrm{C}$. After cooling, the MDA-TBA complex was extracted with $5 \mathrm{ml} n$-butanol and pyridine mixed solution, centrifuged at $1660 \times \boldsymbol{g}$ for $10 \mathrm{~min}$, and the absorbance of the supernatants was read at $532 \mathrm{~nm}$.

Observation of Erythema Formation by UV Irradiation Fur on the dorsal skin of guinea pigs was cut by hair clippers and shavers and dirt on the skin was washed off. About $24 \mathrm{~h}$ later, a gel-like microemulsion prepared with $8.3 \%$ AEROSIL R972 containing genistein was applied to the right side portion of the skin, while a gel-like microemulsion without genistein was applied to the left side. After about $20 \mathrm{~h}$, microemulsion gel remaining on the skin surface was wiped off with wet gauze repeatedly. After drying the skin surface for $4 \mathrm{~h}$, both sides of portion of guinea pig dorsal skin were irradiated for $24 \mathrm{~min}$ by UV light at an intensity of $138 \mu \mathrm{W} / \mathrm{cm}^{2}$, measured with UV-Meter 340 (Ando Keiki Co., Tokyo, Japan). GL20SE UV-B lamps (Sankyo Denki Co., Tokyo), emitting a continuous light spectrum between 280 and $380 \mathrm{~nm}$ with a peak emission at $306 \mathrm{~nm}$, were used. Erythema formation was evaluated using photographs as well as skin redness measurement with color reader CR-13 (Konica Minolta Holdings Inc. (Tokyo, Japan)).

Statistical Analysis Data were analyzed using the Krusk1-Wallis test. Individual differences between medians were examined using Dunn's multiple comparison test.

Acknowledgements This work was supported by a grant from The Cosmetology Research Foundation, Tokyo, Japan. We thank Dr. Hitoshi Masaki and Dr. Yuri Okano, Nikkol Group Cosmos Technical Center Co. for instruction in the technique for the UV irradiation experiment.

\section{References}

1) Kim J., Hwang J.-S., Cho Y.-K., Han Y., Jeon Y.-J., Yang K.-H., Skin Pharmacol. Appl. Skin Physiol., 14, 11-19 (2001).

2) Caddeo C., Teskac K., Sinico C., Kristl J., Int. J. Pharm., 363, 183191 (2008).

3) Vayalil P. K., Elmets C. A., Katiyar S. K., Carcinogenesis, 24, 927936 (2003).

4) Montenegro L., Carbone C., Maniscalco C., Lambusta D., Nicolosi G., Ventura C. A., Puglisi G., Int. J. Pharm., 336, 257-262 (2007).

5) Marti-Mestres G., Mestres J. P., Bres J., Martin S., Ramos J., Vian L., Int. J. Pharm., 331, 139-144 (2007).

6) Fang J.-Y., Hwang T.-L., Huang Y.-L., Fang C.-L., Int. J. Pharm., 310, 131-138 (2006).

7) Pappinen S., Urtti A., "Percutaneous Penetration Enhancers," 2nd ed., ed. by Smith E. W., Maibach H. I., CRC, Boca Raton, 2006, pp. 109116.

8) Heuschkel S., Goebel A., Neubert R. H. H., J. Pharm. Sci., 97, 603631 (2008).

9) Vicentini F. T. M. C., Simi T. R. M., Del Ciampo J. O., Wolga N. O., Pitol D. L., Iyomasa M. M., Bently M. V. L. B., Fonseca M. J. V., Eur. J. Pharm. Biopharm., 69, 948-957 (2008).

10) Kitagawa S., Tanaka Y., Tanaka M., Endo K., Yoshii A., J. Pharm. Pharmacol., 61, 855-860 (2009).

11) Kang S., Chung J. H., Lee J. H., Fisher G. J., Wan Y. S., Duell E. A., Voorhees J. J., J. Invest. Dermatol., 120, 835-841 (2003).

12) Casagrande R., Georgetti S. R., Verri Jr. W.A., Borin M. F., Lopez R. F. V., Fonseca M. J. V., Int. J. Pharm., 328, 183-190 (2007).

13) Kurihara-Bergstrom T., Woodworth M., Feisullin S., Beall P., Lab. Anim. Sci., 36, 396-399 (1986).

14) Date A. A., Nagarsenker M. S., Int. J. Pharm., 355, 19-30 (2008).

15) Cao H., Liu Q., Shi J., XiaonJ., Xu M., Anal. Lett., 41, 521—532 (2008).

16) Delgado-Charro M. B., Iglesias-Vilas G., Blanco-Mendez J., LopezQuintela M. A., Marty J. P., Guy R. H., Eur. J. Pharm. Biopharm., 43, 37- 42 (1997).

17) Debacq-Chainiaux F., Borlon C., De Hertogh B., Remacle J., Morvan P.-Y., Vallee R., Toussaint O., J. Pharm. Pharmacol., 58, 1577-1583 (2006).

18) Casagrande R., Georgetti S. R., Verri Jr. W. A., Dorta D. J., dos Santos A. C., Fonseca M. J. V., J. Photochem. Photobiol. B, 84, 21-27 (2006).

19) Fujii M., Yamanouchi S., Hori N., Iwanaga N., Kawaguchi N., Matsumoto M., Biol. Pharm. Bull., 20, 249-254 (1997).

20) Lee P. J., Langer R., Shastri V. P., Pharm. Res., 20, $264-269$ (2003).

21) Kitagawa S., Kasamaki M., Chem. Pharm. Bull., 54, 242-244 (2006).

22) Kitagawa S., Sakamoto H., Tano H., Chem. Pharm. Bull., 52, 9991001 (2004). 